\title{
Ideophone-gesture composites: depictive type, sensory class, and modality
}

\author{
Janis B. Nuckolls, Tod Swanson, Alex Rice, Diana Sun and Sarah Hatton*
}

LSA Annual Meeting, Portland, Oregon, January 7-11, 2015

1.Introduction. Our paper seeks to understand how ideophones and gestures are interrelated in the Pastaza dialect of Ecuadorian Quichua, hereafter referred to as Pastaza Quichua or simply PQ. Ideophones are a class of expressions found throughout the worlds' languages. They are of interest to linguists because of their fascinating structural features, their complex semantics, and their discourse properties, which draw interlocutors into a shared experience of simulated perceptions. As Dingemanse (2014:387) has stated: "they show rather than tell and depict rather than describe". We consider them depictive because they are uttered not for the purpose of referring, but with the intention of simulating sensory perceptions.

To signal their depictive status, ideophones have special prosodic features built within them. They are often louder, softer, higher pitched, lower pitched, pronounced more slowly, or more quickly than their surrounding utterances. Besides their prosodic marking, forthcoming work by Nuckolls, Stanley, Nielsen and Hopper (2016) has also revealed interesting differences between ideophones' sounds and the sounds of the prosaic or nonideophonic lexicon. The obstruent sounds are increased among PQ ideophones by a number of more complex sounds, including many newly palatalized sounds, expressively aspirated sounds, and a few sounds that fill gaps in the inventory. By contrast, the sonorant sounds of PQ are overwhelmingly underrepresented among ideophones.

The question suggested by these findings, then, which has motivated the present study is the following: Why, given the increased complexity of their sounds and their prosodic elaboration, do PQ speakers invest further in ideophones with gestural depiction as well? We focus in particular, on gestures because a number of researchers have stated that ideophones and gestures have a special affinity with each other, particularly in narrative discourse (Dingemanse 2013:144-146). To approach this question we compiled over 150 ideophone-gesture composite examples from dozens of different contexts, which added up to more than 7 hours of recordings.

2. General Findings. One general finding from our work is that, despite claims about a special relationship between ideophones and gesture, in Pastaza Quichua at least, ideophones do

\footnotetext{
*Janisnnuckolls@byu.edu.Tod.swanson@asu.edu; arrozito21@gmail.com; diana.sun39@gmail.com; sarah@henryshammock.com
} 
not have a monopoly on gestural expression. PQ speakers seem to gesture constantly whether they're using ideophones or not. Two exceptions to this generalization are found in song and in reported discourse. We've witnessed an absence of gestures during the singing of songs. In reported discourse, gestures are used sparingly at best. Putting aside these two contexts of song and reported speech, PQ speakers gesture abundantly during all kinds of discourse.

We've observed, moreover, that there is a slightly greater variety of gestures used in nonideophonic discourse than in ideophonic discourse. If we adopt McNeill's 1992 typology of gesture use, we find every one of his major categories of gesture used in non-ideophonic discourse, namely iconic, metaphoric, deictic and beat gestures, as well as emblems and pantomime. However, we have not seen deictic gestures or emblems co-occurring with PQ ideophones, although other researchers have (Dingemanse 2013, Mihas 2013, Reiter 2011.)

Regarding the relationship between gestures that do occur with ideophones, we may also state another general finding: the occurrence of ideophones with gestures, and the types of gestures with which they are used, doesn't seem to be particularly sensitive to any particular genre of discourse. Rather, it's complexly interdependent upon a number of factors, namely, the sensory class of the ideophone, the semantic concreteness or abstractness of the ideophone, the semantics of the verb it occurs with, its discourse functions, and a speaker's perspective on what is being depicted.

3. Specific Findings. The sensory class of an ideophone seems to be a most promising predictor of whether or not that ideophone will be accompanied by a gesture. Ideophones depictive of visually observable motion are most likely to be accompanied by an iconic gesture that is obviously affiliated with that ideophone's meaning (examples 1-4).

\section{Yaku patca tsaxxxx}

Water just IDEO

'And the water just( went') tsaxxxx'(swiping gesture concurrent with tsax)

When ideophones are depictive of motion and sound, however, there may be a gesture or not. In such instances, speakers may choose to focus only on the sounds and not on their accompanying movements, depending on what a speaker wishes to emphasize.

When ideophones are primarily sound-imitative they may occur with no gesture, with a minimal beat gesture or with a semantically unaffiliated gesture. Example 2 below illustrates an ideophonic depiction of a bird's repeated chirping sounds. After each fourth repetition, the 
speaker lifts his hand up in the air and quickly lowers it, to mark the stretch of repetitions as a unit:

2. tcik t6ik t6ik t6ik

IDEO IDEO IDEO IDEO

(It goes) t6ik t6ik t6ik t6ik ...(hand lifts up and quickly lowers after $4^{\text {th }}$ repetition)

Another finding is that although many PQ ideophones are indeed linked with sensory perceptions, some are rather abstract and their abstractness makes them suitable for gesturing the motions of their accompanying verbs, thus tying the ideophone and the verb together into a unified expression. The ideophone tas, for example may be coarticulated with different gestures, each of which is semantically affiliated with a different verb to express manner of motion, path of motion, and aspectual values such as completiveness and ongoingness. Example 3 below illustrates its use with a gesture that depicts a complete drinking of a substance:

3.tas upi-ra-ni

IDEO drink-PAST-1SG

'I drank it (down) tas' (hand moves downward)

In example 4, the same ideophone is used to describe the way an illness completely left the speakers body, which is gesturally depicted with a hand moving away from her body:

4.tas rimid $^{\mathrm{j}} \mathrm{u} \quad$ sina- $\mathrm{Ka}$

IDEO medicine like-LIM

'(It made the illness disappear) tas (completely) just like medicine.' (with hand moving away from body).

The meaning of the ideophone tas is so abstract that it can collocate with over 40 different verbs.

Finally, even ideophones that are very sensorily grounded may be accompanied by gestures with varying degrees of iconicity that seem linked with gradations of speaker-internal perspective. The ideophone polan for example, depicts rise of an object or living thing from underwater to the surface. It may be articulated with both hands moving up or simply with one hand moving up. We concur, therefore with the insight of Krauss, Chen, and Gottesman (2000:276) that gestural iconicity is a matter of degree.

4. Conclusion. We return to our original question about why speakers invest so much energy into ideophonic performances. We believe that the reasons are both linguistic and cultural. From a linguistic perspective, it seems clear that imageic forms of communication are integrated into the structure of this language. In some instances there is a very straightforward 
semantic relationship between ideophones and gestures, especially when they depict visually observable motion. In other instances, it's necessary to consider not only the interrelations between ideophones and gestures, but between ideophones, verbs and gestures, because the ideophone and its gesture are specifying the manner of a verb's motion, or an image of the verb's action as ongoing, or accomplished and complete. Gestures, therefore, not only illus trate ideophones' meanings, but also tie those meanings together with a verb's grammatical and semantic specifications.

One unresolved question arising from this work is the matter of the relationship between gesture and intonation. Does one drive the other or are they somewhat independent? Intonational foregrounding must also be considered from a cultural perspective because of the tendency for Quichua speakers to emphasize the emotional and sentimental qualities of their perceptions by means of intonation. As a class of expressions, ideophones seem well-positioned to reward future research into the language and culture as well as the language and gesture complex.

\section{References}

Dingemanse, Mark. 2013. Ideophones and gesture in everyday speech. Gesture, 13, 143-165.

doi:10.1075/gest.13.2.02din

Dingemanse, M. (2014). Making new ideophones in Siwu: Creative depiction in conversation. Pragmatics and Society, 5(3), 384-405. doi:10.1075/ps.5.3.04din.

Krauss, Chen, and Gottesman (2000) Lexical Gestures and Lexical Access: A Process Model. In: David McNeill (Ed.), Language and gesture.pp. 261-283). New York: Cambridge University Press.

McNeill, David. 1992. Hand and Mind. Chicago: The University of Chicago Press.

Mihas, Elena. 2013. Gesture, vol. 13:1, pp. 28-62.Composite ideophone-gesture utterances in the Ashéninka Perené ‘community of practice', an Amazonian Arawak society from Central-Eastern Peru. Gesture, vol. 13:1, pp. 28-62.

Nuckolls, Janis, Stanley, Joseph, Nielsen, Elizabeth, and Hopper, Roseanna.2016 The systematic stretching and contracting of ideophonic phonology in Pastaza Quichua. International Journal of American Linguistics (expected, January, 2016).

Reiter, Sabine. 2011. Ideophones in Aweti. Doctoral Dissertation. University of Kiel. 
